\title{
Perfil etiológico de las infecciones urinarias y patrón de sensibilidad de los uropatógenos
}

\author{
J.M. Eiros Bouza ${ }^{\mathrm{a}}$, C. Ochoa Sangrador ${ }^{\mathrm{b}}$ y Grupo Investigador del Proyecto \\ aServicio de Microbiología. Hospital Clínico Universitario. Valladolid. \\ ${ }^{\mathrm{b} C o o r d i n a d o r}$ Servicio de Pediatría. Hospital Virgen de la Concha. Zamora. España.
}

\begin{abstract}
Se presenta una revisión sobre el perfil etiológico de las infecciones urinarias en la infancia y el patrón de sensibilidad de los uropatógenos en nuestro país. Escherichia coli sigue siendo el principal agente etiológico de infección urinaria en la infancia, por lo que su patrón de sensibilidad va a determinar habitualmente la elección terapéutica empírica. El predominio de $E$. coli se ve reducido en ciertas circunstancias, en las que otros microorganismos incrementan su presencia. No obstante, la información clínica disponible al diagnóstico no permite predecir con seguridad la etiología; tan sólo la tinción y el examen microscópico de la orina pueden ayudar a seleccionar el tratamiento. $E$. coli presenta en España un alto porcentaje de resistencia a ampicilina y cotrimoxazol, mientras que mantienen una elevada sensibilidad las cefalosporinas de segunda y tercera generación, fosfomicina, aminoglucósidos y amoxicilina-ácido clavulánico. En algunas áreas, amoxicilina-ácido clavulánico y las cefalosporinas de primera generación presentan elevada resistencia, lo que puede comprometer su uso empírico.

\section{Palabras clave:}

Infección del tracto urinario. Niño. Medicina basada en la evidencia. Conferencia de Consenso. Etiología. Resistencia bacteriana a drogas.
\end{abstract}

\section{ETIOLOGICAL PROFILE OF URINARY TRACT INFECTIONS AND ANTIMICROBIAL SUSCEPTIBILITY OF URINARY PATHOGENS}

$A$ review on the etiological profile of urinary tract infections in childhood and the sensitivity pattern of urinary pathogens in Spain is presented. Escherichia coli continues to be the main etiological agent of urinary tract infection in childhood. Consequently, its sensitivity pattern will usually determine the choice of empirical therapy. The predominance of $E$. coli is reduced in certain circumstances, in which the presence of other microorganisms is increased.
However, the clinical information available at diagnosis does not allow accurate identification of the etiology; only staining and microscopic urine examination can help in treatment selection. In Spain, $E$. coli presents a high percentage of resistance to ampicillin and cotrimoxazole, whereas second- and third-generation cephalosporins, fosfomycin, aminoglycosides and amoxicillin-clavulanate maintain high sensitivity. In some areas, amoxicillin-clavulanate and first-generation cephalosporins show high levels of resistance, which can limit their empirical use.

\section{Key words:}

Urinary tract infections. Child. Evidence-based medicine. Consensus development conference. Etiology. Bacterial drug resistance.

\section{INTRODUCCIÓN}

Como ya se ha comentado en otros documentos, a pesar de que, a diferencia de la edad adulta, en la infancia es habitual la realización de estudios microbiológicos ante la sospecha de infecciones del tracto urinario (ITU), el tratamiento inicial de las ITU es frecuentemente empírico, según etiología más probable y su perfil teórico de sensibilidad a antimicrobianos, por lo que se debe ajustar en función del resultado de los estudios microbiológicos.

Por lo tanto, la sensibilidad de los principales uropatógenos en España va a condicionar la elección de tratamiento empírico. En este documento revisamos el perfil etiológico observado en distintas series de pacientes pediátricos con ITU, así como la sensibilidad que los uropatógenos aislados en esas series han presentado a los principales antimicrobianos.

En la tabla 1 se presenta la distribución de frecuencias por microorganismos, con indicaciones del lugar, época y

Financiado parcialmente por una beca de la Gerencia Regional de Salud de la Junta de Castilla y León (año 2006).

Correspondencia: Dr. C. Ochoa Sangrador.

Unidad de Investigación. Hospital Virgen de la Concha.

Avda. Requejo, 35. 49029 Zamora. España.

Correo electrónico: cochoas@meditex.es 


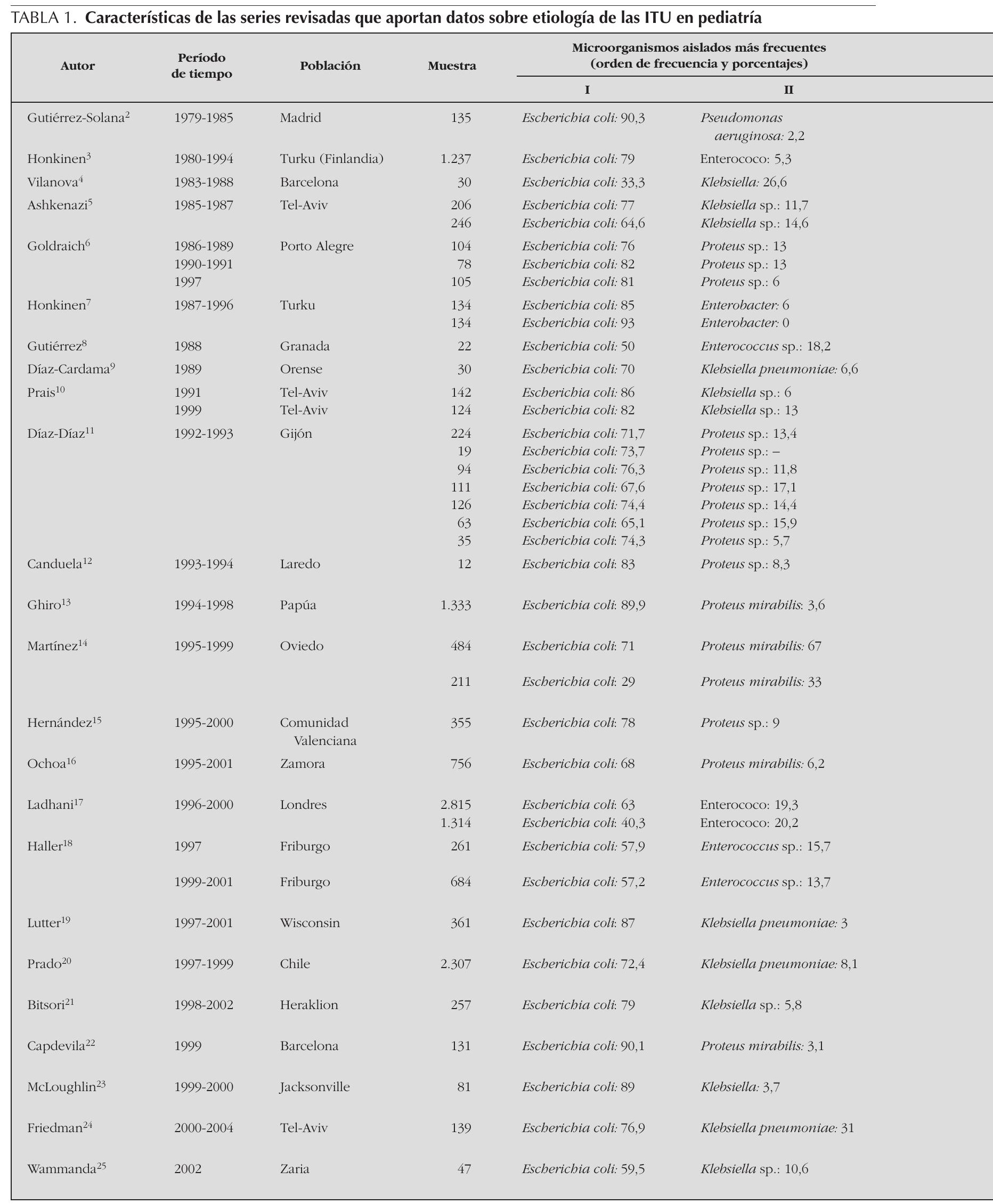

ITU: infecciones del tracto urinario. 
Microorganismos aislados más frecuentes

III

Staphylococcus epidermidis: 2,2

Klebsiella sp.: 4,4

Enterobacter: 16,6

Proteus sp.: 9

Proteus sp.: 9,3

Klebsiella sp.: 8

Klebsiella sp.: 1

Klebsiella sp.: 7

Estafilococo: 6

Estafilococo: 1

Candida albicans: 9,1

Proteus mirabilis: 6,6

Otros 8

Otros 5

Enterococo: 7,2

Enterococo: 15,8

Enterococo: 4,3

Enterococo: 8,1

Enterococo: 4

Enterococo: 12,7

Enterococo: 8,6

Klebsiella sp.: 8,3

Klebsiella oxytoca: 2,1

Klebsiella

pneumoniae: 73

Klebsiella

pneumoniae: 27

Klebsiella 4

pneumoniae:

Pseudomonas

aeruginosa: 4,2

Proteus sp.: 5,8

Pseudomonas sp.: 10,8

Proteus sp.: 9,2

Pseudomonas aeruginosa: 7

Pseudomonas

aeruginosa: 2

Proteus mirabilis: 6,1

\section{Enterococcus}

faecalis: 5,4

Klebsiella

pneumoniae: 3,1

Enterococcus sp.: 3,7

Pseudomonas

aeruginosa: 21

Enterobacter sp.: 10,6 (orden de frecuencia y porcentajes)

IV

V

Procedencia y características de la muestra

Muestras urinarias de 18 niñas con criterios

de ITU recurrentes

Muestras de pacientes hospitalizados

Urinocultivos positivos de 28 recién nacidos hospitalarios

ITU comunitarias

ITU hospitalarias

Muestras de pacientes de urgencias con ITU febril Muestras de pacientes de urgencias con ITU febril Muestras de pacientes de urgencias con ITU febril

Otros: 4

Otros: 6

Klebsiella pneumoniae: 4 Klebsiella pneumoniae: 6

Enterococcus faecalis: 3 Enterococcus faecalis: 2

ITU con bacteriemia

ITU $\sin$ bacteriemia

Proteus mirabilis: 4,5

Pacientes hospitalizados

Pacientes con ITU intrahospitalaria y extrahospitalaria Pacientes con ITU intrahospitalaria y extrahospitalaria

Enterobacter: 1,8

Enterobacter: 5,3

Enterobacter: 1,1

Enterobacter: 1,8

Enterobacter: 2,4

Enterobacter: 1,6

Enterobacter

Pseudomonas: 2,2

Pseudomonas:

Pseudomonas: 2,1

Pseudomonas: 2,7

Pseudomonas: 2,4

Pseudomonas:

Pseudomonas: 5,7

Enterococo: 1,3

aeruginosa: 1,4

Otras enterobacter: 69

Pseudomonas aeruginosa: 58

Otras enterobacter: 31

Pseudomonas

aeruginosa: 42

Pseudomonas

aeruginosa: 3

Enterobacter cloacae: 3

Enterobacter: 3

Streptococcus agalactiae: 2,8

Klebsiella sp.: 3,3

Klebsiella sp.: 7,6

Pseudomonas aeruginosa: 8,4

Proteus sp.: 5,9

Enterococcus sp.: 2

Klebsiella oxytoca: 4,8

Pseudomonas aeruginosa: 3,2

Proteus mirabilis: 4,7

Enterobacter cloacae: 2,3

Citrobacter: 1,2

Proteus sp.: 1,2

Enterobacter: 16

Citrobacter sp.: 9
Global de niños con ITU hospitalizados

Neonatos

Lactantes

Primera ITU

Reinfección simple (menos de 4 al año)

Reinfección frecuente (4 o más infecciones anuales)

Pacientes que acceden por urgencias y permanecen hospitalizados

Hospitalizados con pielonefritis

Hospitalarios y extrahospitalarios con ITU menores de 2 años

Hospitalarios y extrahospitalarios con ITU mayores de 2 años

Hospitalarios y extrahospitalarios

Urinocultivos positivos de pacientes hospitalarios y extrahospitalarios

Aislados urinarios de la comunidad

Aislados urinarios de niños con patología renal

Urinocultivos positivos de pacientes intrahospitalarios y extrahospitalarios

Urinocultivos positivos de pacientes intrahospitalarios y extrahospitalarios

Pacientes hospitalarios con ITU y pielonefritis

Urinocultivos positivos de pacientes hospitalarios y ambulatorios correspondientes a 11 hospitales

Pacientes hospitalizados con primera ITU

Lactantes previamente sanos hospitalarios con primera ITU

Urinocultivos positivos pediátricos de la base de datos del hospital

Niños hospitalizados que cumplían criterios para diagnóstico de ITU

Urinocultivos positivos de pacientes intrahospitalarios y extrahospitalarios
Mayores de 2 años 


\begin{tabular}{|c|c|c|c|c|c|c|c|c|c|c|c|c|c|}
\hline \multirow{2}{*}{ Autor } & \multirow{2}{*}{ Año } & \multicolumn{10}{|c|}{ Porcentaje de sensibilidad a antibióticos } & \multirow{2}{*}{$\begin{array}{c}\text { Tipo } \\
\text { de ITU* }\end{array}$} & \multirow{2}{*}{$\begin{array}{l}\text { Procedencia } \\
\text { de la muestra }\end{array}$} \\
\hline & & AMP & AMC & C1G & C2G & C3G & NIT & GM & TMP-SMX & NAL & FF & & \\
\hline \multirow[t]{2}{*}{ Fritzsche $^{26}$} & $1980-1991$ & 67 & 83 & - & - & - & - & - & 84 & - & - & $c, 4$ & \multirow[t]{2}{*}{ Suiza } \\
\hline & $2000-2003$ & 49 & 89 & 83 & 98,7 & 100 & - & - & 75 & - & - & c, 4 & \\
\hline \multirow[t]{2}{*}{ Ashkenazi ${ }^{5}$} & $1985-1987$ & 53 & - & 76 & 99 & 99 & 99 & 100 & 64 & 99 & - & a & \multirow{2}{*}{$\begin{array}{l}\text { Tel-Aviv } \\
\text { Tel-Aviv }\end{array}$} \\
\hline & $1985-1987$ & 17 & & 38 & 92 & 99 & 89 & 97 & 45 & 95 & - & $\mathrm{d}$ & \\
\hline \multirow[t]{3}{*}{ Goldraich $^{6}$} & 1986-1989 & - & - & - & 65 & - & 92 & - & 40 & 85 & - & c & \multirow{3}{*}{$\begin{array}{l}\text { Porto Alegre } \\
\text { Porto Alegre } \\
\text { Porto Alegre }\end{array}$} \\
\hline & $1990-1991$ & - & - & - & 54 & - & 95 & - & 85 & 92 & - & c & \\
\hline & 1997 & - & - & - & 81 & - & 94 & - & 40 & 95 & - & c & \\
\hline \multirow[t]{2}{*}{ Prais ${ }^{10}$} & 1991 & 37 & - & 90 & - & - & 100 & - & 59 & 98 & - & $\mathrm{b}$ & \multirow{2}{*}{$\begin{array}{l}\text { Israel } \\
\text { Israel }\end{array}$} \\
\hline & 1999 & 32 & 78 & 64 & - & 99 & 98 & - & 67 & 99 & - & $\mathrm{b}$ & \\
\hline Díaz-Díaz ${ }^{11}$ & $1992-1993$ & 39,4 & 90,6 & 57,5 & 99,4 & 100 & - & 100 & 61,9 & - & - & c & Gijón \\
\hline Allen ${ }^{27}$ & 1992-1994 & 55 & - & 97,1 & - & 99,9 & 98,2 & 97,1 & 68,6 & - & - & c & Ontario \\
\hline Ghiro $^{13}$ & 1994-1998 & - & 97 & - & - & 99,9 & - & 98,5 & 92 & - & - & c, 4 & Padua*** \\
\hline Martínez $z^{14}$ & 1995-1999 & 49 & - & 92 & 97 & 100 & 95 & 96 & 82 & - & - & c & Oviedo \\
\hline Hernández ${ }^{15}$ & $1995-2000$ & 20 & 85 & 80 & 95 & 99 & 90 & 95 & 65 & - & 99 & c & Comunidad Valenciana \\
\hline Ochoa $^{16}$ & $1995-2001$ & 36,7 & 93,3 & 95 & 99,3 & 100 & 94,4 & 96,6 & 77,3 & - & 100 & c & Zamora $^{* * *}$ \\
\hline \multirow[t]{2}{*}{ Ladhani $^{17}$} & $1996-2000$ & 49 & 86,4 & 98,5 & 99,9 & - & 94 & 99,9 & 70 & - & - & $\mathrm{a}$ & \multirow[t]{2}{*}{ Londres** } \\
\hline & & 40,8 & 89,4 & 93 & 94 & - & 63,3 & 98,5 & 49,6 & - & - & 4 & \\
\hline \multirow[t]{2}{*}{ Haller $^{18}$} & 1997 & 66 & - & - & 98 & 98 & & & 60 & - & - & $\mathrm{b}$ & \multirow[t]{2}{*}{ Friburgo } \\
\hline & 1999-2001 & 65 & - & - & 99 & 99 & - & - & 60 & - & - & $\mathrm{b}$ & \\
\hline \multirow[t]{2}{*}{ Prado $^{20}$} & 1997-1999 & 23 & 78 & 83 & - & 100 & - & 99 & 44 & & - & c & \multirow[t]{2}{*}{ Chile $^{* * *}$} \\
\hline & & 23 & 84 & 74 & - & 100 & - & 98 & 48 & - & - & $\mathrm{a}$ & \\
\hline Andreu ${ }^{* * * 28}$ & 2002 & 41,3 & 90,8 & - & - & 95,8 & 94,3 & - & 66,1 & - & 98 & b, 3,4 & España**** \\
\hline Marcus $^{29}$ & 2001-2002 & 38 & 92 & 91 & 91 & 99 & 97 & 100 & 62 & 96 & - & c & Tel-Aviv*** \\
\hline Wammanda ${ }^{25}$ & 2002 & 15 & 60 & - & - & 0 & & 80 & 16,7 & - & - & $\mathrm{b}$ & Zaria (Nigeria) \\
\hline
\end{tabular}

*a: ITU en pacientes extrahospitalarios; b: ITU en pacientes hospitalizados y extrahospitalarios; c: ITU en pacientes hospitalizados; d: ITU adquiridas en el hospital; 1: sin factores de riesgo; 2: con factores de riesgo (ingreso previo o exposición previa a antibióticos); 3: ITU no complicadas; 4: ITU complicadas.

${ }^{* *}$ Estudios pediátricos con porcentajes de resistencia a ciprofloxacino inferiores a $5 \%$.

${ }^{*}$ ****Este estudio incluye adultos ( 1.198 cepas de Escherichia coli; el 10,4\% de la población es menor de 20 años). Sensibilidad a ciprofloxacino, $77 \%$. AMC: amoxicilina-ácido clavulánico; AMP: ampicilina; C1G, C2G, C3G: cefalosporina de primera, segunda y tercera generaciones; FF: fosfomicina; GM: gentamicina; NAL: ácido nalidíxico; NIT: nitrofurantoína; TMP-SMX: cotrimoxazol; itu: infecciones del tracto urinario.

características de los pacientes incluidos en cada una. En las tablas de la 2 a la 6 se presentan los porcentajes de sensibilidad de E. coli, Proteus spp., Klebsiella spp., Enterococcus spp. y Pseudomonas aeruginosa. En el análisis de sensibilidad se han tenido en cuenta algunas series españolas de población general (incluidos adultos y niños), ya que permiten obtener estimaciones más precisas. A continuación, se resumen los principales hallazgos de las series pediátricas incluidas en las tablas de la 1 a la 6 , y se comentan también algunos datos de una revisión previamente publicada por nuestro grupo ${ }^{1}$.

- Escherichia coli sigue siendo el principal agente etiológico de ITU en la infancia (70-90\% de los casos), por lo que su patrón de sensibilidad va a determinar habitualmente la elección terapéutica empírica.

- El predominio de E. coli se ve reducido en ciertas circunstancias en las que otros microorganismos incrementan su presencia. La exposición previa a antibióticos, el antecedente de hospitalización o la existencia de anomalías urinarias incrementan la probabilidad de que otros microorganismos, como Proteus mirabilis, Klebsiella spp. y Pseudomonas aeruginosa, sean los agentes responsables de ITU. Debemos considerar también, especialmente en el niño pequeño, Enterococcus faecalis. Otros microorganismos poco frecuentes son: Enterobacter cloacae, Streptococcus agalactiae, Staphylococcus spp., Serratia marcescens, Morganella morganii, Citrobacter spp. y Acinetobacter spp. La información clínica disponible al diagnóstico no permite predecir con seguridad la etiología; tan sólo la tinción y el examen microscópico de la orina pueden ayudar a seleccionar un determinado tratamiento. Esta exploración complementaria, habitualmente no disponible, debería considerarse en pacientes con ITU de alto riesgo.

- E. coli presenta en España un alto porcentaje de resistencia a ampicilina y cotrimoxazol, por lo que estos antibióticos no resultan adecuados como tratamientos empíricos, a pesar de que la resistencia a cotrimoxazol se ha reducido algo en los últimos años. Entre los antibióticos que en España mantienen una elevada actividad frente a $E$. coli, se encuentran las cefalosporinas de se- 


\begin{tabular}{|c|c|c|c|c|c|c|c|c|c|c|c|c|c|}
\hline \multirow{2}{*}{ Autor } & \multirow{2}{*}{ Año } & \multicolumn{10}{|c|}{ Porcentaje de sensibilidad a antibióticos } & \multirow{2}{*}{$\begin{array}{c}\text { Tipo } \\
\text { de ITU* }\end{array}$} & \multirow{2}{*}{$\begin{array}{l}\text { Procedencia } \\
\text { de la muestra }\end{array}$} \\
\hline & & AMP & AMC & C1G & C2G & C3G & NIT & GM & TMP-SMX & NAL & FF & & \\
\hline Ashkenazi ${ }^{5}$ & $\begin{array}{l}1985-1987 \\
1985-1987\end{array}$ & $\begin{array}{r}25 \\
9\end{array}$ & & $\begin{array}{l}50 \\
31\end{array}$ & $\begin{array}{r}100 \\
87\end{array}$ & $\begin{array}{l}100 \\
100\end{array}$ & $\begin{array}{l}63 \\
65\end{array}$ & $\begin{array}{r}100 \\
96\end{array}$ & $\begin{array}{l}75 \\
48\end{array}$ & $\begin{array}{l}88 \\
83\end{array}$ & $\begin{array}{l}- \\
-\end{array}$ & $\begin{array}{l}\mathrm{a} \\
\mathrm{d}\end{array}$ & $\begin{array}{l}\text { Tel-Aviv } \\
\text { Tel-Aviv }\end{array}$ \\
\hline Díaz-Díaz ${ }^{11}$ & $1992-1993$ & 50 & 96,7 & 86,7 & 93,3 & 96,7 & - & 100 & 56,7 & - & - & c & Gijón \\
\hline Ghiro $^{13}$ & $1994-1998$ & - & 93 & - & - & 89 & - & 100 & 80 & - & - & c, 4 & Ontario \\
\hline Martínez ${ }^{14}$ & 1995-1999 & 75 & - & 100 & 100 & 100 & 100 & 100 & 86 & - & 86 & c & Oviedo \\
\hline Hernández ${ }^{15}$ & $1995-2000$ & 40 & 40 & 80 & 90 & 95 & 25 & 85 & 60 & - & - & c & Comunidad Valenciana \\
\hline Ochoa $^{16}$ & $1995-2001$ & 60,5 & 100 & 93 & 100 & 100 & 0 & 100 & 74,4 & - & 83 & c & Zamora \\
\hline Ladhani $^{17}$ & $1996-2000$ & $\begin{array}{l}84,8 \\
80\end{array}$ & $\begin{array}{l}95,7 \\
90\end{array}$ & $\begin{array}{l}98,7 \\
96\end{array}$ & $\begin{array}{l}98 \\
90\end{array}$ & $\begin{array}{l}- \\
-\end{array}$ & $\begin{array}{l}0 \\
0\end{array}$ & $\begin{array}{l}99,4 \\
64\end{array}$ & $\begin{array}{l}64 \\
74,5\end{array}$ & - & - & $\begin{array}{l}\mathrm{a} \\
4\end{array}$ & Londres \\
\hline Andreu $^{* * 28}$ & 2002 & 61,6 & 93,9 & - & - & 96,4 & 0 & - & 62,1 & - & 76 & b, 3,4 & España*** \\
\hline
\end{tabular}

"a: ITU en pacientes extrahospitalarios; b: ITU en pacientes hospitalizados y extrahospitalarios; c: ITU en pacientes hospitalizados; d: ITU adquiridas en el hospital; 1: sin factores de riesgo; 2: con factores de riesgo (ingreso previo o exposición previa a antibióticos); 3: ITU no complicadas; 4: ITU complicadas. **Este estudio incluye adultos (196 cepas; el 10,4\% de la población es menor de 20 años). Sensibilidad a ciprofloxacino, $83 \%$.

AMC: amoxicilina-ácido clavulánico; AMP: ampicilina; C1G, C2G, C3G: cefalosporina de primera, segunda y tercera generaciones; FF: fosfomicina; GM: gentamicina; NAL: ácido nalidíxico; NIT: nitrofurantoína; TMP-SMX: cotrimoxazol; itu: infecciones del tracto urinario.

\section{TABLA 4. Sensibilidad a los principales antibióticos de Klebsiella spp. en distintas series pediátricas}

\begin{tabular}{|c|c|c|c|c|c|c|c|c|c|c|c|c|c|}
\hline \multirow{2}{*}{ Autor } & \multirow{2}{*}{ Año } & \multicolumn{10}{|c|}{ Porcentaje de sensibilidad a antibióticos } & \multirow{2}{*}{$\begin{array}{c}\text { Tipo } \\
\text { de ITU* }\end{array}$} & \multirow{2}{*}{$\begin{array}{l}\text { Procedencia } \\
\text { de la muestra }\end{array}$} \\
\hline & & AMP & AMC & C1G & C2G & C3G & NIT & GM & TMP-SMX & NAL & FF & & \\
\hline \multirow[t]{2}{*}{ Ashkenazi ${ }^{5}$} & $1985-1989$ & 33 & & 75 & 100 & 100 & 92 & 100 & 63 & 100 & & a & Tel-Aviv \\
\hline & $1985-1989$ & 31 & & 61 & 97 & 97 & 92 & 97 & 33 & 97 & & $\mathrm{~d}$ & Tel-Aviv \\
\hline \multirow[t]{2}{*}{ Prais ${ }^{10}$} & 1991 & 1 & - & 50 & & - & 89 & - & 67 & 100 & & $\mathrm{~b}$ & Israel \\
\hline & 1999 & 7 & 78 & 71 & & 78 & 73 & - & 78 & 93 & & $\mathrm{~b}$ & Israel \\
\hline Ghiro $^{13}$ & $1994-1998$ & - & 100 & - & - & 93 & - & 100 & 82 & - & & c, 4 & Ontario \\
\hline Martínez ${ }^{14}$ & 1995-1999 & - & - & & 77 & 50 & 50 & 50 & 47 & - & 37 & c & Oviedo \\
\hline Hernández ${ }^{15}$ & $1995-2000$ & 0 & 0 & 75 & 85 & 100 & 60 & 90 & 75 & - & & c & Comunidad Valenciana \\
\hline Ochoa $^{16}$ & $1995-2001$ & - & 90,9 & 72,7 & 86,4 & 95,5 & 90 & 95,5 & 100 & - & 88 & c & Zamora \\
\hline \multirow[t]{2}{*}{ Ladhani $^{17}$} & $1996-2000$ & 0 & 97,8 & 98 & 98,9 & - & 100 & 88,7 & 74,5 & - & & $\mathrm{a}$, & Londres \\
\hline & & 0 & 90 & 95 & 89 & - & 90 & 58 & 48,9 & - & & 4 & \\
\hline \multirow[t]{2}{*}{ Prado $^{20}$} & 1997-1999 & 0 & 34 & 31 & - & 37 & - & 41 & 34 & - & & c & Chile \\
\hline & & 3 & 52 & 41 & - & 77 & - & 85 & 72 & - & & $\mathrm{a}$ & \\
\hline Andreu**28 & 2002 & 0 & 94,5 & - & - & 97,7 & 67,1 & - & 93,2 & - & 78 & b, 3,4 & España*** \\
\hline Wammanda 25 & 2002 & 25 & 80 & - & - & 100 & - & 50 & 20 & - & & & Zaria (Nigeria) \\
\hline
\end{tabular}

*a: ITU en pacientes extrahospitalarios; b: ITU en pacientes hospitalizados y extrahospitalarios; c: ITU en pacientes hospitalizados; d: ITU adquiridas en el hospital; 1: sin factores de riesgo; 2: con factores de riesgo (ingreso previo o exposición previa a antibióticos); 3: ITU no complicadas; 4: ITU complicadas.

**:Este estudio incluye adultos (147 cepas; el 10,4\% de la población es menor de 20 años). Sensibilidad a ciprofloxacino, $93 \%$.

AMC: amoxicilina-ácido clavulánico; AMP: ampicilina; C1G, C2G, C3G: cefalosporina de primera, segunda y tercera generaciones; FF: fosfomicina; GM: gentamicina;

NAL: ácido nalidíxico; NIT: nitrofurantoína; TMP-SMX: cotrimoxazol; itu: infecciones del tracto urinario.

gunda y tercera generaciones, fosfomicina, aminoglucósidos y amoxicilina-ácido clavulánico. No obstante, la combinación amoxicilina-ácido clavulánico presenta en algunas áreas porcentajes de resistencia crecientes. Igualmente, la resistencia a las cefalosporinas de primera generación es muy variable, y ha ascendido en algunas áreas hasta niveles que pueden comprometer su uso empírico.

- Los datos disponibles sobre fluoroquinolonas, de series de aislamientos de población general, sugieren que se ha producido un importante aumento de la resistencia de E. coli a ciprofloxacino y otras fluoroquinolonas. Las escasas series pediátricas que analizan estos antibióticos muestran mínimos porcentajes de resistencia. No obstante, a pesar de que se está extendiendo el uso de estos antimicrobianos en la infancia, por el momento ha quedado limitado al tratamiento de ITU complicadas, dirigido por cultivo y antibiograma.

- Proteus mirabilis tiene un perfil de sensibilidad similar al de E. coli, aunque en algunas series de urocultivos de población general y pediátrica ha mostrado una menor sensibilidad a fosfomicina. Klebsiella pneumoniae 


\begin{tabular}{|c|c|c|c|c|c|c|c|c|c|c|}
\hline \multirow{2}{*}{ Autor } & \multirow{2}{*}{ Año } & \multicolumn{7}{|c|}{ Porcentaje de sensibilidad a antibióticos } & \multirow{2}{*}{$\begin{array}{c}\text { Tipo } \\
\text { de ITU* }\end{array}$} & \multirow{2}{*}{$\begin{array}{l}\text { Procedencia } \\
\text { de la muestra }\end{array}$} \\
\hline & & AMP & AMC & VAN & TEI & CIP & GM & TMP-SMX & & \\
\hline Díaz-Díaz ${ }^{11}$ & $1992-1993$ & 100 & 100 & - & - & - & - & 12,5 & c & Gijón \\
\hline Ghiro $^{13}$ & 1994-1998 & - & 100 & - & - & - & 63 & 60 & c, 4 & Ontario \\
\hline Hernández ${ }^{15}$ & $1995-2000$ & 100 & 100 & - & - & - & - & 30 & c & Comunidad Valenciana \\
\hline Ochoa $^{16}$ & 1995-2001 & 100 & 100 & - & - & - & - & - & c & Zamora \\
\hline Ladhani ${ }^{17}$ & $1996-2000$ & $\begin{array}{l}99 \\
68,5\end{array}$ & $\begin{array}{l}99 \\
68\end{array}$ & $\begin{array}{l}100 \\
93,6\end{array}$ & - & $\begin{array}{l}0,4 \\
0,8\end{array}$ & $\begin{array}{l}0,4 \\
0,8\end{array}$ & $\begin{array}{l}90,8 \\
42,2\end{array}$ & $\begin{array}{l}\mathrm{a} \\
4\end{array}$ & Londres \\
\hline
\end{tabular}

"a: ITU en pacientes extrahospitalarios; b: ITU pacientes hospitalizados y extrahospitalarios; c: ITU en pacientes hospitalizados; d: ITU adquiridas en el hospital; 1: sin factores de riesgo; 2: con factores de riesgo (ingreso previo o exposición previa a antibióticos); 3: ITU no complicadas; 4: ITU complicadas. AMC: amoxicilina-ácido clavulánico; AMP: ampicilina; CIP: ciprofloxacino; GM: gentamicina; ITU: infecciones del tracto urinario; TEI: teicoplanina; TMP-SMX: cotrimoxazol; VAN: vancomicina.

\section{TABLA 6. Sensibilidad a los principales antibióticos de Pseudomonas aeruginosa en distintas series pediátricas} y de adultos nacionales

\begin{tabular}{|c|c|c|c|c|c|c|c|c|c|c|c|}
\hline \multirow{2}{*}{ Autor } & \multirow{2}{*}{ Año } & \multicolumn{8}{|c|}{ Porcentaje de sensibilidad a antibióticos } & \multirow{2}{*}{$\begin{array}{c}\text { Tipo } \\
\text { de ITU* }\end{array}$} & \multirow{2}{*}{$\begin{array}{l}\text { Procedencia } \\
\text { de la muestra }\end{array}$} \\
\hline & & P-T & CAZ & CPM & IP & NAL & CIP & GM & AK & & \\
\hline \multirow[t]{2}{*}{ Ashkenazi $^{5}$} & 1985-1989 & 100 & 100 & & & 33 & & 100 & & a & Tel-Aviv \\
\hline & 1985-1989 & 75 & 92 & & & 17 & & 83 & & $\mathrm{~d}$ & Tel-Aviv \\
\hline Hernández ${ }^{15}$ & $1995-2000$ & & 100 & & & & & 75 & 95 & $\mathrm{c}$ & Comunidad Valenciana \\
\hline Ghiro $^{13}$ & 1994-1998 & & 80 & & & & 83 & 81 & & c, 4 & Ontario \\
\hline Haller $^{18}$ & 1997 & 82 & 82 & & 100 & & & & & $\mathrm{~b}$ & Friburgo \\
\hline Wammanda 25 & 2002 & & & & & & & 50 & & & Zaria (Nigeria) \\
\hline Ochoa $^{16}$ & 1995-2001 & & & & & & & 96,4 & & c & Zamora \\
\hline Atienza $^{* * 30}$ & 1997 & & 97,0 & & & & 85,0 & 89,0 & 90,0 & $\mathrm{~b}$ & Hellín** (Albacete) \\
\hline Queipó Zaragoza**31 & 1998 & & & & & & 85,0 & & & $\mathrm{~b}$ & Valencia** $^{* *}$ \\
\hline Alonso $^{* * 32}$ & 1999 & & 66,0 & & & & 62,0 & 84,0 & 94,0 & $\mathrm{~b}$ & España*** \\
\hline Daza**33 $^{*}$ & 1999 & 88,0 & 88,0 & 76,0 & 85,0 & & 68,0 & 85,0 & 100,0 & a & Granada** $^{* *}$ \\
\hline Colomina*34 & 2000 & & 93,0 & & & & 61,0 & 96,0 & 100,0 & $\mathrm{a}$ & Elda $^{* * *}$ \\
\hline
\end{tabular}

*a: ITU en pacientes extrahospitalarios; b: ITU en pacientes hospitalizados y extrahospitalarios; c: ITU en pacientes hospitalizados; d: ITU adquiridas en el hospital; 1: sin factores de riesgo; 2: con factores de riesgo (ingreso previo o exposición previa a antibióticos); 3: ITU no complicadas; 4: ITU complicadas.

${ }^{*}$ Estos estudios incluyen población general española.

AK: amikacina; CAZ: ceftazidima; CIP: ciprofloxacino; CPM: cefepime; GM: gentamicina; IP: imipenem; ITU: infecciones del tracto urinario; NAL: ácido nalidíxico;

P-T: piperacilina/tazobactam

presenta resistencia natural a ampicilina, manteniendo una alta sensibilidad a otros antibióticos habitualmente activos frente a ella. La aparición de cepas de Klebsiella productoras de betalactamasas de espectro ampliado puede explicar que algunas series, fundamentalmente con casuística hospitalaria, muestren un descenso importante de la sensibilidad a cefalosporinas. Pseudomonas aeruginosa mantiene una buena sensibilidad a carbapenémicos (imipenem y meropenem), piperacilina-tazobactam, ceftazidima, tobramicina y amikacina, mientras que ha descendido algo para ciprofloxacino y gentamicina.

- En la elección de tratamiento empírico debemos considerar que aunque las cefalosporinas de segunda y tercera generación presentan un perfil discretamente mejor que amoxicilina-ácido clavulánico para enterobacterias, sólo amoxicilina-ácido clavulánico permite cubrir un eventual, aunque poco frecuente, Enterococcus faecalis.
Asimismo, tenemos que evaluar que el antecedente de antibioterapia, hospitalización o anomalías urinarias incrementa el riesgo de que el agente etiológico sea resistente a los antibióticos de uso habitual.

- Por último, teniendo en cuenta que ningún antibiótico garantiza una cobertura al 100\% de todos los posibles microorganismos, en casos de ITU de alto riesgo podemos necesitar asociaciones de antibióticos. Para cubrir un posible enterococo, tendremos que incluir en la asociación ampicilina. Por otra parte, si queremos cubrir Pseudomonas aeruginosa conviene que asociemos antibióticos específicos, ya que combinaciones clásicas, que incluyan gentamicina, pueden resultar insuficientes. Finalmente, resulta excepcional en la infancia tener que recurrir a carbapenémicos con el propósito de cubrir posibles microorganismos multirresistentes (cepas de Klebsiella con betalactamasas de espectro ampliado, Acinetobacter o Pseudomonas). 


\section{Miembros del Grupo Investigador del Proyecto "Estudio de la Variabilidad e Idoneidad del Manejo Diagnóstico y Terapéutico de las Infecciones del Tracto Urinario en la Infancia"}

C. Ochoa Sangrador (Hospital Virgen de la Concha, Zamora); C. Pérez Méndez y G. Solís Sánchez (Hospital de Cabueñes, Gijón, Asturias); J.C. Molina Cabañero y J. Lara Herguedas (Hospital del Niño Jesús, Madrid); F. Conde Redondo, R. Bachiller Luque, J.Ma Eiros Bouza, V. Matías del Pozo y B. Nogueira González (Hospital Clínico Universitario, Valladolid); M. ${ }^{a}$ C. Urueña Leal, J.M. Andrés de Llano, P. Rostami y J.F. Sáenz Martín (Complejo Asistencial de Palencia); E. Formigo Rodríguez, M. Cueto Baelo y M.`C. González Novoa (Hospital Xeral-Cies, Vigo, Pontevedra); N. Silva Higuero y H. González García (Hospital de Medina del Campo, Valladolid); J. González de Dios (Hospital de Torrevieja, Alicante); M. Brezmes Raposo (Hospital La Fe, Valencia); M.̣.M. Urán Moreno y J.M. Gea Ros (Hospital Universitario San Juan, Alicante); Mํㅡ. V. Barajas Sánchez, Mํㅜ.F. Brezmes Valdivieso y MํaA. Fernández Testa (Hospital Virgen de la Concha, Zamora)

\section{Bibliografía}

1. Ochoa Sangrador C, Eiros Bouza JM, Méndez CP, Inglada Galiana L, y Grupo de Estudio de los Tratamientos Antibioticos. Etiología de las infecciones del tracto urinario y sensibilidad de los uropatógenos a los antimicrobianos. Rev Esp Quimioter. 2005;18:124-35.

2. Gutiérrez-Solana LG, Pozo J, Cano J, Molina JC, Sánchez Bayle M, Ecija JL, et al. Infeccion recurrente del tracto urinario en niñas. An Esp Pediatr. 1987;26:11-4.

3. Honkinen O, Lehtonen OP, Ruuskanen O, Huovinen P, Mertsola J. Cohort study of bacterial species causing urinary tract infection and urinary tract abnormalities in children. BMJ. 1999;318:770-1.

4. Vilanova Juanola JM, Canos Molinos J, Rosell Arnold E, Figueras Aloy J, Comas Masmitja LL, Jiménez González R. Infección del tracto urinario en el recién nacido. An Esp Pediatr. 1989; 31:105-9.

5. Ashkenazi S, Even-Tov S, Samra Z, Dinari G. Uropathogens of various childhood populations and their antibiotic susceptibility. Pediatr Infect Dis J. 1991;10:742-6.

6. Goldraich NP, Manfroi A. Febrile urinary tract infection: Escherichia coli susceptibility to oral antimicrobials. Pediatr Nephrol. 2002;17:173-6.

7. Honkinen O, Jahnukainen T, Mertsola J, Eskola J, Ruuskanen O. Bacteremic urinary tract infection in children. Pediatr Infect Dis J. 2000;19:630-4.

8. Gutiérrez J, Alados JC, Liebana J, Martin A. Estudio comparativo entre la flora aerobia del aparato genital y la causante de infecciones urinarias en niños menores de siete años. Acta Pediatr Esp. 1989;47:159-62.

9. Díaz-Cardama I, Blanco M, Seara J, Salgado P, Pereira P, González M, et al. Infecciones urinarias en la infancia. Aten Primaria. 1989;6:133

10. Prais D, Straussberg R, Avitzur Y, Nussinovitch M, Harel L, Amir J. Bacterial susceptibility to oral antibiotics in community acquired urinary tract infection. Arch Dis Child. 2003;88: 215-8.

11. Díaz-Díaz E, Solís Sánchez G, Viejo De La Guerra G, Cuervo Valdés JJ, Fernández Menéndez JM, Matesanz Pérez JL. Estudio de la sensibilidad in vitro de los agentes etiológicos de la infección urinaria del niño. Rev Esp Pediatr. 1993; 49:487-90.

12. Canduela Martínez V, Mongil Ruiz I, Cagigas Daza P, Docio Nieto $\mathrm{S}$. Tratamiento de la infección urinaria en lactantes con ceftriaxona. Rev Esp Pediatr. 1995;51:341-3.

13. Ghiro L, Cracco AT, Sartor M, Comacchio S, Zacchello G, Dall'Amico R. Retrospective study of children with acute pyelonephritis. Evaluation of bacterial etiology, antimicrobial susceptibility, drug management and imaging studies. Nephron. 2002;22:8-15

14. Martínez Suárez V, Cimadevilla Suárez R, Amil Pérez B, Ordóñez Álvarez FA, Pérez Castro S, Santos Rodríguez F, et al. Patrón de sensibilidad a antimicrobianos en uropatógenos aislados en niños. Rev Esp Quimioterap. 2001;14:63-8.

15. Hernández Aguado I, Lumbreras B, Vioque J. Evaluación de la tira reactiva para el diagnóstico de infección urinaria en niños y adultos. Med Clin. 2001;117:116-7.

16. Ochoa Sangrador C, Santos Fernández MI, Brezmes Valdivieso MF, Marugán Isabel VM, García Mangas MJ, Carrascal Tejado A. Tendencias en la sensibilidad a antimicrobianos de los uropatógenos en la infancia (1995-2001). Bol Pediatr. 2004;44: 3-8.

17. Ladhani S, Gransden W. Increasing antibiotic resistance among urinary tract isolates. Arch Dis Child. 2003;88:444-5.

18. Haller M, Brandis M, Berner R. Antibiotic resistance of urinary tract pathogens and rationale for empirical intravenous therapy. Pediatr Nephrol. 2004;19:982-6.

19. Lutter SA, Currie ML, Mitz LB, Greenbaum LA. Antibiotic resistance patterns in children hospitalized for urinary tract infections. Arch Pediatr Adolesc Med. 2005;159:924-8.

20. Prado V, Trucco O, Duran C, Mamani R, Royer M. Perfil de resistencia a los antimicrobianos en agentes causantes de infección del tracto urinario en niños chilenos. Programa de vigilancia PRONARES. Rev Med Chil. 2001;129:877-85.

21. Bitsori M, Maraki S, Raissaki M, Bakantaki A, Galanakis E. Community-acquired enterococcal urinary tract infections. Pediatr Nephrol. 2005;20:1583-6.

22. Capdevila Cogul E, Martín Ibáñez I, Mainou Cid C, Toral Rodríguez E, Cols Roig M, Agut Quijano T, et al. Primera infección urinaria en el lactante sano: epidemiología y pautas de diagnóstico y tratamiento. An Esp Pediatr. 2001;55:310-4.

23. McLoughlin TG, Jr., Joseph MM. Antibiotic resistance patterns of uropathogens in pediatric emergency department patients. Acad Emerg Med. 2003;10:347-51

24. Friedman S, Reif S, Assia A, Levy I. Clinical and laboratory characteristics of non-E. coli urinary tract infections. Arch Dis Child. 2006;91:845-6.

25. Wammanda RD, Aikhionbare HA, Ogala WN. Use of nitrite dipstick test in the screening for urinary tract infection in children. West Afr J Med. 2000;19:206-8.

26. Fritzsche M, Ammann RA, Droz S, Bianchetti MG, Aebi C. Changes in antimicrobial resistance of Escherichia coli causing urinary tract infections in hospitalized children. Eur J Clin Microbiol Infect Dis. 2005;24:233-5.

27. Allen UD, MacDonald N, Fuite L, Chan F, Stephens D. Risk factors for resistance to "first-line" antimicrobials among urinary tract isolates of Escherichia coli in children. CMAJ. 1999;160: 1436-40.

28. Andreu A, Alós JI, Gobernado M, Marco F, De la Rosa M, García-Rodríguez JA. Grupo Cooperativo Español para el Estudio de la Sensibilidad Antimicrobiana de los Patógenos Urinarios. Etiología y sensibilidad a los antimicrobianos de los uropatógenos causantes de la infección urinaria baja adquirida en la comunidad. Estudio nacional multicéntrico. Enferm Infecc Microbiol Clin. 2005;23:4-9. 
29. Marcus N, Ashkenazi S, Yaari A, Samra Z, Livni G. Non-Escherichia coli versus Escherichia coli community-acquired urinary tract infections in children hospitalized in a tertiary center: Relative frequency, risk factors, antimicrobial resistance and outcome. Pediatr Infect Dis J. 2005;24:581-5.

30. Atienza Morales MP, Castellote Varona FJ, Romero Portilla C. Infección urinaria y sensibilidad antibiótica en el sur de la provincia de Albacete. An Med Interna. 1999;16:236-8.

31. Queipó-Zaragoza JA, Budia Alba A, Mascaros García E, Gómez-Ferrer Lozano A, Gobernado Serrano M, Jiménez Cruz JF. Evolución de la resistencia microbiana a fluorquinolonas en un hospital terciario. Actas Urol Esp. 2000;24: 381-7.
32. Alonso R, Fernández-Aranguiz A, Colom K, Herreras A, Cisterna R. Perfil de aislamientos bacterianos y sensibilidad antimicrobiana. Estudio multicéntrico mediante corte de un día. Rev Esp Quimioterap. 2000;13:384-93.

33. Daza R, Gutiérrez J, Piédrola G. Antibiotic susceptibility of bacterial strains isolated from patients with community-acquired urinary tract infections. Int J Antimicrob Agents. 2001;18: $211-5$.

34. Colomina Avilés J, Fuentes Luri S, Cascales Ramos P, Pascual Pérez R, Cabo Moya C. Infección urinaria y resistencia bacteriana en urocultivos de pacientes ambulatorios. An Med Interna. 2000;17:506-7. 